\title{
EFEITOS DA UMIDADE DO SOLO E DA COBERTURA VEGETAL NA DISTRIBUIÇÃO E ABUNDÂNCIA DE DROSERA MONTANA (DROSERACEAE)
}

\author{
Mário M. Espírito-Santo ${ }^{1}$ \\ Márcio S. Werneck ${ }^{2}$
}

\section{Recebido em 14/04/1999. Aceito em 01/10/1999}

\begin{abstract}
RESUMO - (Efeitos da umidade do solo e da cobertura vegetal na distribuiçâo e abundância de Drosera montana (Droseraceae)). Este trabalho teve como objetivo comparar o padrão de distribuição, abundância e tamanho de Drosera montana em microhabitats sob diferentes condições de umidade do solo e cobertura vegetal na Serra do Cipó, MG. Foi analisado um transecto de $15 \times 3 \mathrm{~m}$, no qual foram mapeados e medidos todos os indivíduos encontrados, e calculados os indices de agregação de Clark \& Evans e de Hopkins. O transecto foi dividido em 45 quadrados de $1 \mathrm{~m}^{2}$, nos quais foram coletadas amostras de solo e medidas suas percentagens de umidade. A percentagem de cobertura vegetal foi estimada pelo método de Daumbemirre para cada quadrado. D. montana apresentou padrão de distribuição agregado. A maior abundância de indivíduos ocorreu em locais de umidade e cobertura vegetal intermediárias, em um contínuo de condições ambientais variando de locais de solo seco com pouca cobertura vegetal a locais de solo muito úmido com muita cobertura. Entretanto, o tamanho de $D$. montana não parece ser afetado pela umidade ou cobertura vegetal, sendo provavelmente determinado pelo sucesso de captura de presas. Estes resultados sugerem que o padrão agregado observado para esta espécie está relacionado à disponibilidade de água e luz, mas seu crescimento é possivelmente influenciado pela interação entre fatores bióticos e abióticos.
\end{abstract}

Palavras-chave - relação planta-solo, agregação, distribuição espacial, planta carnívora, Serra do Cipó

ABSTRACT - (Effects of soil humidity and vegetation cover on the distribution and abundance of Drosera montana
(Droseraceae)). This study aimed at comparing the distribution, abundance and size of Drosera montana in microhabitats
under different conditions of soil humidity and vegetation cover in Serra do Cipó, state of Minas Gerais. In a transect of
$15 \times 3 \mathrm{~m}$, all individuals of $D$. montana were mapped and their diameters were measured. We calculated the aggregation
indexes of Clark \& Evans and of Hopkins. The transect was divided into 45 quadrats of $1 \mathrm{~m}^{2}$. Soil samples were collected
in each quadrat for moisture content determination. The percentage of vegetation cover was estimated for each quadrat
using the method of Daumbemirre. $D$. montana presented an aggregated pattern of distribution. A higher abundance of
individuals was observed on sites with intermediate soil humidity and vegetation cover, in a continuum of environmental
conditions varying from sites with dry soils and low vegetation cover to sites with wet soils and high vegetation cover.
However, the size of $D$. montana was apparently not affected by soil humidity or vegetation cover, and is probably
determined by prey trapping success. These results suggest that the aggregated pattern observed for this species is
related to water and light availability, but its growth is possibly influenced by an interaction of biotic and abiotic factors.

Key words - plant-soil relation, aggregation, spatial distribution, carnivorous plant, Serra do Cipó

1 Departamento de Biologia Geral, ICB, Universidade Federal de Minas Gerais, C. Postal 486, CEP 30161-970, Belo Horizonte, MG, Brasil.e-mail: esanto@mono.icb.ufmg.br

2 Departamento de Botânica, ICB, Universidade Federal de Minas Gerais, C. Postal 486, CEP 30161-970, Belo Horizonte, MG, Brasil 


\section{Introdução}

Plantas carnívoras são aquelas capazes de capturar presas, absorver seus metabólitos e utilizá-los em seu crescimento e desenvolvimento (Aldenius et al. 1983). Estes organismos possuem ampla distribuição geográfica, estando representados em quase todas as regiões do mundo, mas são restritos exclusivamente a habitats pobres em nutrientes (Lüttge 1983; Benzing 1987). A disponibilidade de nutrientes é considerada, de forma geral, o principal fator abiótico que limita a distribuição de plantas carnívoras (Zamora et al. 1998). Entretanto, a maioria dos estudos realizados consiste em experimentos conduzidos em laboratório, tendo como foco predominante a limitação destes organismos por nutrientes, principalmente nitrogênio e fósforo (Zamora $\mathrm{et} \mathrm{al}$. 1998). Desta forma, poucos trabalhos verificaram efeitos de outros fatores ambientais, como água e luz, na abundância de plantas carnívoras em condições naturais (Zamora 1995; Zamora et al. 1998).

Apesar de ocorrerem em habitats pobres em nutrientes, plantas carnívoras freqüentemente ocupam locais úmidos e ensolarados (Lüttge 1983; Givnish et al. 1984; Benzing 1987), indicando que recursos como água e luz também afetam seu desempenho. A intensidade de irradiação solar é, provavelmente, importante fator influenciando a sobrevivência de plantas carnívoras, uma vez que a obtenção de energia ocorre obrigatoriamente através da fotossíntese, extraindo fósforo e nitrogênio suplementares das presas capturadas (Benzing 1986; 1987). A umidade do solo também influencia a distribuição e abundância destes organismos, já que a disponibilidade de água afeta consideravelmente a capacidade fotossíntetica e digestiva (Givnish $e t$ al. 1984). Assim, estudos envolvendo efeitos da umidade do solo e intensidade luminosa sobre plantas carnívoras em condições naturais são necessários para o melhor entendimento da ecologia e evolução dessas espécies.

A qualidade do habitat é determinante do sucesso de uma população (Ricklefs 1990). Em locais de grande heterogeneidade ambiental, é comum a ocorrência de variações nas condições bióticas e abióticas em escalas muito pequenas, levando à formação de microhabitats de maior ou menor qualidade em uma área reduzida (Harper 1977). A quantidade de água no solo pode mudar grandemente em pequenas distâncias, levando a aumento na probabilidade de germinação de sementes e sobrevivência de uma planta em manchas mais favoráveis (Harper 1977; Smith 1984). Além disso, variações na umidade do solo provocam alterações em outros fatores, como disponibilidade de nutrientes (Ricklefs 1990), afetando o desenvolvimento vegetal. Assim, é possível que a abundância de determinada espécie varie em escala local, em resposta à heterogeneidade edáfica.

Este trabalho teve como objetivo verificar como varia a abundância da planta carnívora Drosera montana St. Hil. (Droseraceae) em manchas sob diferentes condições de umidade do solo e de densidade da cobertura vegetal, e quais os efeitos dessa variação no padrão de distribuição espacial da espécie. Especificamente, foram examinadas as seguintes questões: a) Qual o efeito da umidade do solo e da densidade de cobertura vegetal na abundância de $D$. montana? b) Como estes fatores afetam o padrão de distribuição de D. montana? c) Como estes fatores afetam o tamanho de D. montana?

\section{Material e métodos}

Drosera montana é uma planta rasteira, com as folhas dispostas em roseta e adpressas ao solo, predominantemente vermelhas e glandulares (Silva \& Giulietti 1997). O seu porte é reduzido, com o comprimento do caule variando de 0,5 a $1,5 \mathrm{~cm}$, e o comprimento da roseta variando de 0,5 a $3,0 \mathrm{~cm}$. É espécie perene, de ampla distribuição no Brasil, estando representada nas regiões nordeste, centro-oeste, sudeste e sul (Silva e Giulietti 1997).

Área de estudo - Este estudo foi conduzido na Serra do Cipó, localizada na porção sul da cadeia do Espinhaço $\left(19^{\circ} 20^{\prime} \mathrm{S}\right.$ e $\left.43^{\circ} 44^{\prime} \mathrm{W}\right)$, caracterizada por altitudes de 1000 a $1400 \mathrm{~m}$. O substrato rochoso mais comum é o quartzito, onde se desenvolve o campo rupestre. A flora é adaptada às variações extremas da temperatura, baixa 
umidade, ventos fortes, solos rasos e pobres em nutrientes e a alta insolação (Giulietti et al. 1987). A vegetação é tipicamente herbácea e arbustiva, com poucas árvores isoladas em afloramentos rochosos. O clima da região é do tipo mesotérmico (Cwb de Köppen), com a estação seca indo de maio a agosto, e a chuvosa de setembro a abril. A precipitação anual média é de cerca de $1.500 \mathrm{~mm}$.

A área de estudo está localizada a altitude de $1.250 \mathrm{~m}$, em local de campo aberto, com predominância de gramíneas. A quantidade de água existente na parte superficial do solo varia grandemente, indo de solos com saturação de água a solos secos, em curtas distâncias. O mesmo ocorre com a cobertura vegetal, que varia de solos relativamente desnudos (alta penetração de luz solar) a áreas com elevada densidade de gramíneas (pouca penetração de luz solar) em pequena escala espacial. Desta forma, a área é caracterizada por grande heterogeneidade de microhabitats, formados por manchas com diferentes disponibilidades de água e luz.

Coleta de dados - A coleta de dados foi realizada em julho/1998, no meio da estação seca. Para determinar o padrão de distribuição de $D$. montana, foi estabelecido um transecto de $3 \times 15 \mathrm{~m}$, no qual todos os indivíduos foram contados $\mathrm{e}$ mapeados. Posteriormente, foram calculados os índices de agregação de Clark \& Evans (1954) e de Hopkins (1954), com o auxílio de programa próprio para análise de distribuição espacial (desenvolvido em QBASIC por Figueira 1998; sem fins comerciais).

Para verificar a influência da umidade do solo e da cobertura vegetal na abundância de $D$. montana, a área do transecto foi dividida em 45 quadrados de $1 \mathrm{~m}^{2}$, e amostras de solo até $10 \mathrm{~cm}$ de profundidade foram coletadas no centro de cada quadrado com o auxílio de pá. Cem gramas cada amostra foram pesados e secos em estufa a $200^{\circ} \mathrm{C}$, por duas horas. Após a secagem, as amostras foram pesadas novamente para determinação da percentagem de umidade do solo. A percentagem de cobertura vegetal foi estimada visualmente através do método de abundância-cobertura de Daumbemirre (Mueller-Dumbois \& Ellemberg
1974) em cada um dos quadrados. A relação entre a percentagem de cobertura vegetal e a umidade do solo foi determinada através de correlação de Spearman, uma vez que os dados não apresentaram distribuição normal (Zar 1984). A relação entre abundância de indivíduos de D. montana e umidade do solo e cobertura vegetal em cada quadrado foram obtidas através de correlações de Spearman (Zar 1984). Além disso, comparou-se a abundância de indivíduos e a percentagem de cobertura vegetal em cada quadrado ao longo de cinco classes de percentagem de umidade do solo: $<20,20-25,25-30,30-35$ e $>35 \%$. Posteriormente, estas variáveis foram comparadas entre classes através de teste de Kruskal-Wallis, seguido de teste de comparação múltipla entre tratamentos (Zar 1984).

Para verificar a relação entre o tamanho de D. montana e a umidade do solo e a cobertura vegetal, foi medido o diâmetro da roseta de cada indivíduo mapeado dentro do transecto, com o auxílio de paquímetro (precisão de 0,1 milímetro). Foram realizadas regressões lineares entre o tamanho dos indivíduos e a umidade do solo e a percentagem de cobertura vegetal dos quadrados nos quais estes indivíduos se encontravam. Posteriormente, examinou-se a distribuição de diâmetro dos indivíduos nas mesmas cinco classes de percentagem de umidade do solo e comparado entre classes através de teste de Kruskal-Wallis, seguido de teste de comparação múltipla entre tratamentos (Zar 1984). Todos os testes estatísticos foram realizados com o auxílio do software SYSTAT 8.0 for Windows (1998), e as médias são apresentadas com \pm 0 erro padrão.

\section{Resultados}

A percentagem de água no solo apresentou enorme heterogeneidade dentro do transecto, variando de menos de $3 \%$ a mais de $70 \%$ em diferentes quadrados $(\bar{x}=25,8 \pm 2,05 \%)$. O mesmo ocorreu para a percentagem de cobertura vegetal, que variou de $40 \%$ até $100 \%$ em quadrados distintos $(\overline{\mathrm{x}}=71,3 \pm 2,67 \%)$. A abundância de $D$. montana mostrou grande variação, indo de 0 até 20 indivíduos $(\bar{x}=3,19 \pm 0,68)$ em diferentes quadrados do transecto. 


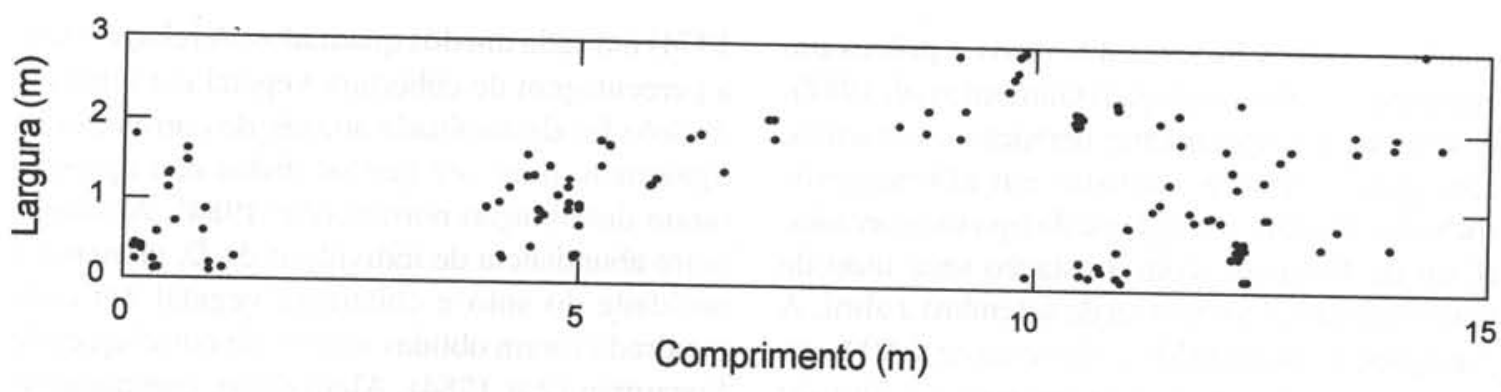

Figura 1. Distribuição espacial de Drosera montana em um transecto de $3 \times 15 \mathrm{~m}$ na Serra do Cipó, MG.

O índice de agregação de Clark \& Evans para a população de $D$. montana foi de $0,38(\mathrm{df}=130$, $\mathrm{z}=-13,5, \mathrm{n}=139$ ), o que indica distribuição espacial fortemente agregada. Este resultado é corroborado pelo índice de Hopkins ( $\mathrm{Ih}=0,86)$. A Fig. 1 ilustra a distribuição das plantas no transecto, mostrando sua ocorrência em manchas.

A percentagem de cobertura vegetal mostrou correlação positiva significativa com a percentagem de umidade do solo $(r=0,56, p<0,0005, n=45$; Fig. 2). Além disso, a percentagem de cobertura vegetal foi significativamente maior em classes de maior umidade do solo (teste de Kruskal-Wallis=17,63, $\mathrm{p}<0,001$; Tab. 1). O número de indivíduos de $D$. montana por quadrado não mostrou correlação linear significativa com a umidade do solo $(\mathrm{r}=0,185$, $\mathrm{p}>0,05, \mathrm{n}=45)$ e com a cobertura vegetal $(\mathrm{r}=-0,093$, $\mathrm{p}>0,05, \mathrm{n}=45$ ). Drosera montana apresentou tendência a maior abundância em valores intermediários de percentagem de umidade e de cobertura vegetal (Fig. 3 a, 3b). Apesar da abundância de indivíduos de $D$. montana ter sido maior nas 3 classes intermediárias de percentagem de umidade (Tab. 1), só houve diferença estatisticamente significativa entre a abundância das duas primeiras classes de umidade (teste de KruskalWallis=6,81, $d f=4, p>0,05$; Tab. 1).
O tamanho dos indivíduos de $D$. montana variou bastante, indo de 0,35 a $2,6 \mathrm{~cm}$ diâm. $(\bar{x}$ $=1,9 \pm 0,3 \mathrm{~cm}$ ). Não houve relação linear entre o diâmetro dos indivíduos e a umidade do solo $\left(r^{2}=0,003, p>0,05, n=139\right)$ e a cobertura vegetal $\left(r^{2}=0,006, p>0,05, n=139\right)$. Além disso, o diâmetro dos indivíduos não apresentou diferenças significativas entre classes de umidade (teste de Kruskal-Wallis $=8,07, \mathrm{df}=4, \mathrm{p}>0,05$; Tab. 1). $\mathrm{O}$ tamanho dos indivíduos variou com a umidade e a cobertura vegetal, mas não mostrou a mesma tendência observada para abundância de indivíduos (Fig. 4a, 4b; Tab. 1).

\section{Discussão}

Plantas carnívoras geralmente ocorrem em ambientes de solo úmido, pobre em nutrientes e de grande luminosidade (Lüttge 1983; Benzing 1987), apresentando distribuição bastante restrita. Em escala local, seria esperado que estas plantas se agregassem em microhabitats de solo mais úmido ou de menor cobertura vegetal, onde a disponibilidade de luz é maior. Entretanto, a interação positiva entre umidade do solo e cobertura vegetal proporciona gradiente de condições ambientais que varia de locais de solo muito seco e com pouca

Tabela 1. Percentagem média de cobertura vegetal, número e diâmetro médios ( $\pm \mathrm{SE})$ dos indivíduos de Drosera montana em cinco classes de umidade em um transecto de $3 \times 15 \mathrm{~m}$ na Serra do Cipó, MG. Letras distintas indicam médias estatisticamente diferentes.

\begin{tabular}{ccccccc}
$\begin{array}{c}\text { \% de umidade } \\
\text { do solo }\end{array}$ & $\begin{array}{c}\text { \% média de } \\
\text { cobertura vegetal }\end{array}$ & $\begin{array}{c}N^{\circ} \text { médio de } \\
\text { indivíduos }\end{array}$ & $\begin{array}{c}N^{\mathrm{a}} \text { de } \\
\text { quadrados }\end{array}$ & $\begin{array}{c}\text { Diâm. médio dos } \\
\text { indivíduos (cm) }\end{array}$ & $\begin{array}{c}\text { No. de } \\
\text { indivíduos }\end{array}$ \\
\hline$<20$ & $56,0 \pm 0,54^{\mathrm{a}}$ & $0,5 \pm 0,22^{\mathrm{a}}$ & 10 & $1,42 \pm 0,15^{\mathrm{a}}$ & 5 \\
$20-25$ & $69,2 \pm 0,42^{\mathrm{ac}}$ & $5,75 \pm 1,99^{\mathrm{b}}$ & 12 & $1,14 \pm 0,06^{\mathrm{a}}$ & 69 \\
$25-30$ & $65,6 \pm 0,44^{\text {acd }}$ & $3,78 \pm 1,28^{\mathrm{ab}}$ & 9 & $1,23 \pm 0,06^{\mathrm{a}}$ & 34 \\
$30-35$ & $83,3 \pm 0,56^{\mathrm{bcd}}$ & $3,67 \pm 1,45^{\mathrm{ab}}$ & 6 & $1,10 \pm 0,08^{\mathrm{a}}$ & 22 \\
$>35$ & $90,0 \pm 0,5^{\mathrm{b}}$ & $1,13 \pm 0,48^{\mathrm{ab}}$ & 8 & $1,45 \pm 0,12^{\mathrm{a}}$ & 9 \\
\hline
\end{tabular}


cobertura vegetal (alta penetração de luz) a locais de solo muito úmido e muita cobertura vegetal (baixa penetração de luz).

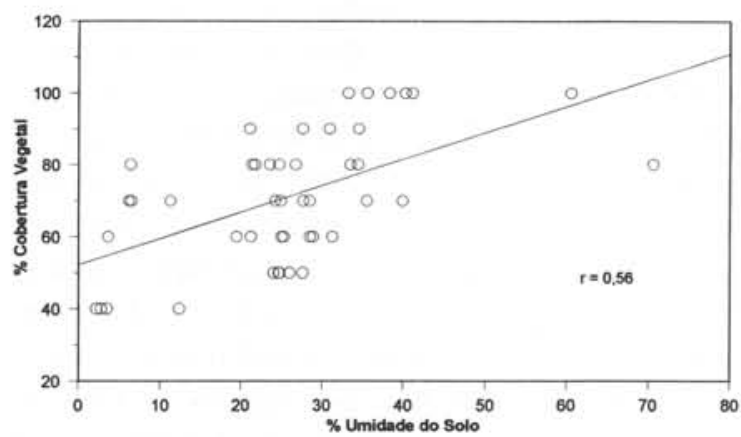

Figura 2. Relaçâo entre percentagem de cobertura vegetal e percentagem de umidade do solo em 45 quadrados de um transecto de $3 \times 15 m$ na Serra do Cipó, MG (correlação de Spearman, $r=0,56$ ).
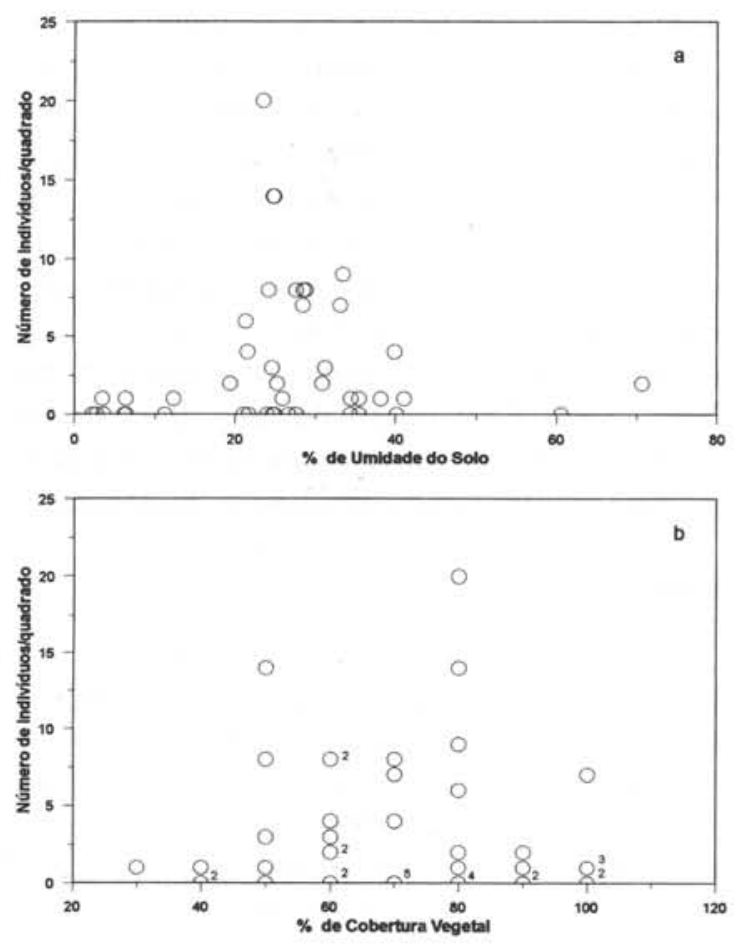

Figura 3. Relação entre a abundância de indivíduos de Drosera montana e a percentagem de umidade do solo (a) e percentagem de cobertura vegetal (b) em 45 quadrados em um transecto de $3 \times 15 \mathrm{~m}$ na Serra do Cipó, MG.

Os números na figura indicam pontos superpostos
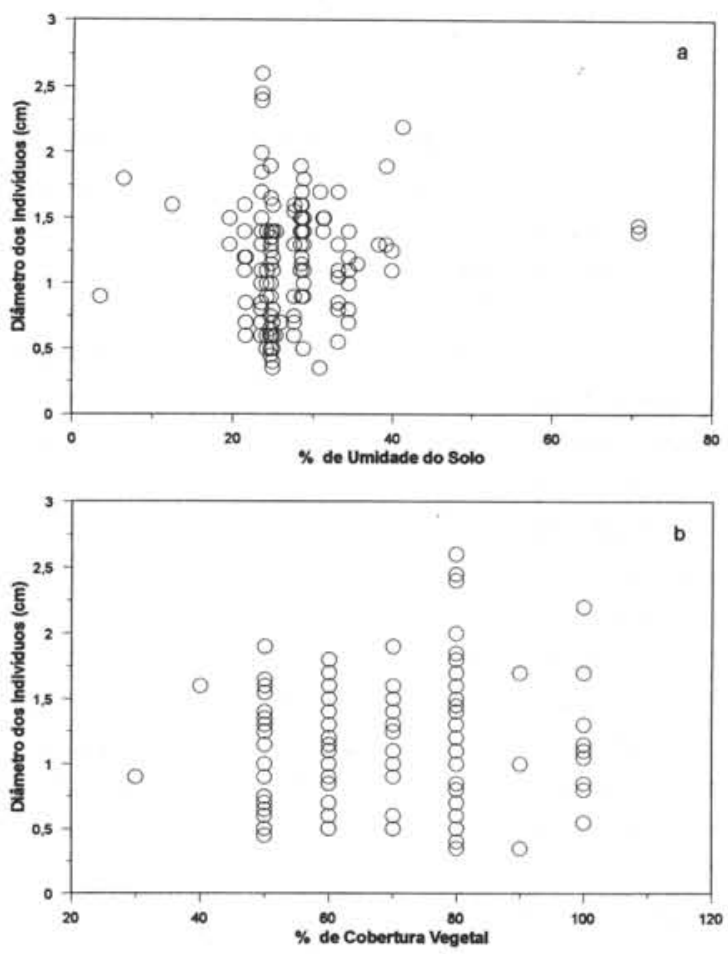

Figura 4. Relação entre o diâmetro da roseta dos indivíduos de Drosera montana e a percentagem de umidade do solo (a) e percentagem de cobertura vegetal (b) em 45 quadrados em um transecto de $3 \times 15 \mathrm{~m}$ na Serra do Cipó, MG.

A maior abundância de indivíduos de $D$. montana tendeu a ocorrer em solos de umidade e cobertura vegetal intermediárias, o que possivelmente reflete sua limitação tanto por disponibilidade de água como por radiação solar. Uma vez que o sistema radicular dessa planta é rudimentar, a escassez de água afeta consideravelmente a absorção deste recurso. Deste modo, a capacidade fotossintética e, conseqüentemente, a sobrevivência da espécie seriam diminuídos em locais de solo seco (Lüttge 1983; Givnish et al. 1984). Em contraste, a anóxia provocada em solos com excesso de água pode limitar o desenvolvimento de $D$. montana, uma vez que não foram descritas nesta espécie estruturas especiais para captação de oxigênio em ambientes encharcados (Silva \& Giulietti 1997). Além disso, em solos muito úmidos, a densidade de cobertura vegetal é mais intensa, diminuindo a disponibilidade de luz para $D$. montana. Como a 
espécie possui porte reduzido e pequena área foliar, é provável que a vegetação circundante influencie de forma determinante seu desempenho. Chander \& Anderson (1976) mostraram que indivíduos de diferentes espécies de Drosera capturando presas no escuro apresentam crescimento reduzido. Desta forma, em ambientes de baixa luminosidade, a carnivoria não constitui vantagem adaptativa, uma vez que a captura de presas supre apenas a demanda de nutrientes necessários para a sobrevivência de espécies com esta síndrome (Givnish et al. 1984; Zamora 1995; Zamora et al. 1998). O carbono obtido de insetos capturados é limitado, e o crescimento de plantas carnívoras depende incondicionalmente da fotossíntese (Lüttge 1983). Assim, em locais de alta disponibilidade de água, a luminosidade é limitante e vice-versa, o que explica a tendência de $D$. montana de se agregar em microhabitats de umidade e cobertura intermediárias, onde a disponibilidade conjunta de luz e água é maior.

Outro fator que pode influenciar a distribuição de $D$. montana é seu mecanismo de dispersão. Em geral, sementes dispersas pelo vento possuem alta capacidade de colonização, podendo percorrer grandes distâncias (Smith 1984; Benzing 1987). Entretanto, apesar de D. montana apresentar dispersão anemocórica, esta espécie apresenta porte reduzido e é rasteira, de forma que seus propágulos possuem baixa dispersibilidade (Benzing 1987). Este fator é, em certos casos, responsável por padrões de distribuição agregada apresentados por espécies dispersas pelo vento (Figueira 1998).

O tamanho de D. montana não foi maior em locais de umidade e cobertura vegetal intermediárias, sem padrão claro de aumento ou diminuição em gradientes de disponibilidade de água e luz. Apesar de plantas carnívoras serem autotróficas e da produção de carbono através da fotossíntese ser fundamental para o crescimento, já foi mostrado que este é bastante estimulado pela captura de presas (Aldenius et al. 1983; Lüttge 1983), havendo correlação positiva entre a fotossíntese potencial máxima e o conteúdo de nitrogênio das folhas dessas plantas (Benzing 1987). Desta forma, é provável que o tamanho dos indivíduos de $D$. montana sofra maior influência direta do sucesso de captura de presas do que da disponibilidade de água e luz.

Segundo Zamora (1995), o sucesso de captura de insetos por uma planta carnívora é resultado de processo complexo, no qual a abundância, a distribuição espaço-temporal e o comportamento das presas determinam a quantidade de biomassa obtida e assimilada. Neste estudo, não foi possível avaliar o sucesso de captura de D. montana e os possíveis efeitos em suas dimensões, abundância e distribuição, devido à escassez de presas capturadas por essa espécie na época do ano em que o estudo foi realizado (apenas um dos 139 indivíduos amostrados havia capturado um díptero). Futuros estudos devem considerar as variações sazonais nas condições bióticas e abióticas do ambiente e suas conseqüências para a dinâmica populacional de $D$. montana.

Assim, o padrão de distribuição e abundância apresentado por $D$. montana é influenciado pela disponibilidade de água e luz, de modo que esta espécie tende a se agregar em manchas nas quais a disponibilidade destes dois fatores conjuntamente é maior. $O$ porte reduzido e rasteiro desta espécie limita a capacidade de competir por água e luz, assim como a capacidade de dispersão, o que provavelmente contribui também para o padrão observado. Entretanto, são necessários maiores estudos sobre a dinâmica populacional desta espécie ao longo do ano, para maior entendimento dos processos bióticos e abióticos que determinam sua distribuição e abundância.

\section{Agradecimentos}

Os Autores agradecem ao IBAMA e aos funcionários do Parque Nacional da Serra do Cipó, pela hospitalidade durante nossa estada no parque; a Maurício Lopes de Faria, Onildo João MariniFilho, Edivani Franceschinelli, Carlos Abraham de Knegt Miranda, Cláudia Jacobi e dois revisores anônimos, pelas valiosas sugestões nas versões iniciais deste manuscrito; ao CNPq, que concedeu bolsas de Mestrado aos Autores. Este trabalho foi financiado pela International Foundation for Science e pelo curso de Pós-Graduação em Ecologia, Conservação e Manejo de Vida Silvestre da Universidade Federal de Minas Gerais. 


\section{Referências bibliográficas}

Aldenius, J.; Carlsson, B. \& Karlsson, S. 1983. Effects of insect trapping on growth and nutrient content of Pinguicula vulgaris $\mathrm{L}$. in relation to the nutrient content of the substrate. New Phytologist 93: 53-60.

Benzing, D. H. 1986. Foliar specialization for animal-assisted nutrition in Bromeliaceae. Pp. 235-256. In: B. E. Juniper \& T. R. E. Southwood (Eds.), Insects and the Plant Surface. Edward Arnold, London.

Benzing, D. H. 1987. The origin and rarity of botanical carnivory. Trends in Ecology and Evolution 2: 364-369.

Chandler, G. E. \& Anderson, J. W. 1976. Studies on the nutrition and growth of Drosera species with reference to the carnivorous habit. New Phytologist 76: 129-141.

Clark, P. J. \& Evans, F. C. 1954. Distance to the nearest neighbor as a measure of spatial relationships in populations. Ecology 35: 445-453.

Figueira, J. E. C. 1998. Dinâmica de populações de Paepalanthus polyanthus (Eriocaulaceae) na Serra do Cipó, MG. Tese de Doutorado. UNICAMP, Campinas.

Giulietti, A. M.; Menezes, N. L.; Pirani, J. R.; Meguro, M. \& Wanderley, M. G. L. 1987. Flora da Serra do Cipó, Minas Gerais: Caracterização geral e lista de espécies. Boletim de Botânica da Universidade de São Paulo 9: 1-151.

Givnish, T. J.; Burkhardt, E. L.; Happel, R. E. \& Weintraub, J. D. 1984. Carnivory in the bromeliad Brocchinia reducta, with a cost/benefit model for the general restriction of carnivorous plants to sunny, moist, nutrient-poor habitats. American Naturalist 124: 479-497.
Hopkins, B. 1954. A new method of determining the type of distribution of plant individuals. Annals of Botany 18: 213-227.

Harper, J. L. 1977. Population biology of plants. Academic Press, New York.

Lüttge, U. 1983. Ecophysiology of carnivorous plants. Pp. 489-517. In: O. L. Lange; P. S. Nobe; C. B. Osmond \& H. Ziegler (Eds.), Physiological plant ecology IIIResponses to the chemical and biological environment, vol. 12C. Springer, New York.

Mueller-Dumbois, D. \& Ellemberg, H. 1974. Aims and methods of vegetation ecology. John Wiley \& Sons, New York.

Ricklefs, R. E. 1990. Ecology. W. H. Freeman \& Company, New York.

Silva, T. R. S. \& Giulietti, A. M. 1997. Levantamento das Droseraceae do Brasil. Boletim de Botânica da Universidade de São Paulo 16: 75-105.

Smith, A. P. 1984. Post dispersal parent-offspring conflict in plants: antecedent and hypothesis from the Andes. American Naturalist 123: 354-370.

SYSTAT 8.0 Statistics. 1998. SYSTAT user's guide. SPSS Inc., Chicago.

Zamora, R. 1995. The trapping success of a carnivorous plant, Pinguicula vallisneriifolia: the cummulative effects of availability, attraction, retention and robbery of prey. Oikos 73: 309-322.

Zamora, R.; Gomez, J. M. \& Hódar, J. A. 1998. Fitness responses of a carnivorous plant in contrasting ecological scenarios. Ecology 79: 1630-1644.

Zar, J. H. 1984. Biostatiscal Analysis. Prentice-Hall, New Jersey. 